\title{
Late cardiotoxicity after low dose of anthracycline therapy for acute lymphoblastic leukemia in childhood
}

\author{
Els Vandecruys • Veerle Mondelaers • Daniel De Wolf • \\ Yves Benoit • Bert Suys
}

Received: 17 February 2011 / Accepted: 16 May 2011 / Published online: 1 June 2011

(C) The Author(s) 2011. This article is published with open access at Springerlink.com

\begin{abstract}
Introduction Late cardiotoxicity is a known complication of anthracycline therapy but the long-term effects of low cumulative doses are not well documented. We studied late cardiotoxicity in survivors of childhood acute lymphoblastic leukemia (ALL) treated with low anthracycline doses 10 to 20 years earlier.

Methods Seventy-seven ALL survivors who received a cumulative anthracycline dose $<250 \mathrm{mg} / \mathrm{m}^{2}$ and were at least 10 years after treatment were evaluated for signs of clinical heart failure. Cardiac function was assessed by echocardiography including tissue Doppler measurements of the septal mitral annulus in 37 ALL survivors 10.618.3 years (median 13.3 years) after anthracycline treatment with cumulative doses of $180(n=19)$ or $240 \mathrm{mg} / \mathrm{m}^{2}(n=18)$. The control group consisted of 30 healthy volunteers matched for age, sex, BSA, and BMI.

Results No clinical relevant cardiotoxicity was found. Left ventricular shortening fraction (SF) was significantly reduced in male ALL survivors. Three of the 19 male ALL survivors had an SF below 30\%. Male ALL survivors showed a significantly lower early filling velocity to atrial contraction velocity ratio but myocardial velocity during
\end{abstract}

E. Vandecruys $(\square) \cdot V$. Mondelaers $\cdot$ Y. Benoit

Department of Pediatric Hemato-Oncology,

Ghent University Hospital,

De Pintelaan 185,

9000 Ghent, Belgium

e-mail: els.vandecruys@ugent.be

D. De Wolf $\cdot$ B. Suys

Department of Pediatric Cardiology, Ghent University Hospital,

Ghent, Belgium early filling was comparable between patients and controls. ALL survivors had a significantly longer isovolumetric relaxation time (IVRT). Thirty percent of the ALL survivors have an abnormal IVRT compared to the normal range of the controls.

Conclusion and implications for cancer survivors At a median of 13.3 years after exposure to cumulative doses of anthracyclines of 180 or $240 \mathrm{mg} / \mathrm{m}^{2}$, no clinical relevant cardiotoxicity was found but subclinical cardiac abnormalities were present in $30 \%$ of the patients.

Keywords Cardiotoxicity after cancer therapy $\cdot A L L \cdot$ Late effects · Anthracycline-related cardiotoxicity

\section{Introduction}

Anthracycline antibiotics, predominantly doxorubicin (DOX) and daunorubicin (DNR), are highly effective in the treatment of childhood malignancies, but are potentially cardiotoxic at all dose ranges. Studies from the early 1990s showed a high incidence of late myocardial damage in previously well long-term survivors of childhood cancer [1-3]. As many as $65 \%$ of acute lymphoblastic leukemia (ALL) survivors showed subclinical abnormalities of left ventricular (LV) systolic function at a mean of 6.4 years after the completion of anthracycline therapy with a cumulative dose of 228 to $550 \mathrm{mg} / \mathrm{m}^{2}$ [2]. More recent reports have shown that the prevalence of cardiac abnormalities is much lower with cumulative anthracycline doses of less than 250-300 mg/m² [4, 5]. No late cardiac damage was demonstrated in childhood ALL survivors at a median of 14.8 years after induction treatment including a cumulative dose of DNR $100 \mathrm{mg} / \mathrm{m}^{2}$, compared to 
survivors who received the same treatment but without DNR[6]. Most reports of late anthracycline cardiotoxicity concern studies of heterogeneous diagnostic groups receiving various anthracycline cumulative doses and having a mean duration of follow-up after anthracycline exposure of less than 10 years.

Serial monitoring of left ventricular function by means of echocardiography (through measurement of the shortening fraction (SF)) has been advocated but LV restriction is a late functional event and SF is also influenced by the loading conditions of the heart. In some previous studies, different indicators of subclinical damage as elevation of afterload (end-systolic stress) [2, 4, 7] and impaired relaxation [7, 8] have been documented, with SF being normal.

Tissue Doppler imaging (TDI) has shown to be very sensitive in identifying subclinical cardiomyopathy in various conditions $[9,10]$.

We report the results of a cardiac evaluation with the use of tissue Doppler imaging of the septal mitral annulus besides standard 2D- and M-mode echocardiography in long-term survivors of childhood ALL at a mean of 13.6 years after anthracycline treatment with a cumulative dose of 180 or $240 \mathrm{mg} / \mathrm{m}^{2}$.

\section{Study population and methods}

Study population

The subjects of the study were long-term survivors of ALL in first complete remission who received a cumulative anthracycline dose $<250 \mathrm{mg} / \mathrm{m}^{2}$ and were at least 10 years after treatment. Patients with Down syndrome or a congenital heart disease were excluded. Eighty-five survivors meeting these criteria were identified from our center-based registry. Seventyseven of them are still attending our long-term follow-up clinic and none of them has signs of clinical heart failure. Thirtyseven patients agreed to participate in the study. All patients were treated in our center according to the EORTC-ALL58831/2 (33 patients) or 58881-protocol (four patients). They received DNR $120 \mathrm{mg} / \mathrm{m} 2$ as part of the induction treatment and DOX 60 or $120 \mathrm{mg} / \mathrm{m}^{2}$ as part of the re-induction treatment. The cumulative dose of cyclophosphamide was 0 or $2 \mathrm{~g} / \mathrm{m}^{2}$ in the low-risk group and $3 \mathrm{~g} / \mathrm{m}^{2}$ in the medium and high risk group. No patient received radiotherapy of the mediastinum. The patients in the low-risk group did not receive prophylactic cranial irradiation, the patients in the medium and high-risk group without CNS involvement at diagnosis were randomized to receive prophylactic cranial irradiation or not. More details about the risk group definition and treatment are found in the article by Vilmer et al. [11].

The control group consisted of 31 healthy volunteers matched for age, sex, and BSA since 11 out of the 37 patients were still in puberty and echocardiographic parameters are increasing with somatic growth (chamber size and wall thickness). They were also matched for BMI because of the potential influence of body composition on cardiac function. These 31 control subjects were chosen out of a larger group of healthy children, adolescents, and young adults who volunteered to undergo the same cardiac evaluation.

The study protocol was approved by the hospital ethical review board.

\section{Methods}

The cardiac evaluation consisted of an interview with attention for cardiac-related problems, measurement of blood pressure, a 12-lead electrocardiogram (ECG) and a complete echocardiographic study with a simultaneous ECG (standard lead II).

\section{Echocardiographic study}

A complete echocardiographic study with a simultaneous ECG (standard lead II) was performed on each patient and control subject by a single observer to avoid interobserver variability. Guided by two-dimensional echocardiography, standard M-mode recordings of the left ventricular dimensions and function were obtained. Left ventricular posterior wall thickness at end systole (LVPWS) and at end diastole (LVPWD) and left ventricular internal dimensions at end systole (LVIDS) and at end diastole (LVIDD) were measured. SF was calculated according to the formula (LVIDD-LVIDS/ LVIDD) $\times 100 \%$. A SF of $<28 \%$ was considered as abnormal. End-systolic wall stress (ESWS) was calculated according to the Rowland formula [12]. The left ventricular mass index and relative posterior wall thickness were calculated according to the formula of Devereux et al. [13]. Conventional Doppler tracings of the mitral and the tricuspid valve and pulmonary venous return flow were obtained from an apical fourchamber view; the Doppler cursor line was placed in the inlet at an angle as parallel to flow as possible and in the position of maximal velocity to measure peak early $(E)$ and peak atrial $(A)$ velocities. $E / A$ ratio, being an indicator of diastolic function, was calculated. Isovolumetric relaxation time (IVRT) was measured as the time interval between the first high-frequency component of the second heart sound (on the phonocardiogram) and the onset of early ventricular filling. Doppler values of the aortic and pulmonary flow were measured from the suprasternal and parasternal short axis respectively. Duration values were corrected for heart rate. Tissue Doppler values of the septal mitral annulus were obtained from the apical four-chamber view, peak early diastolic $\left(E^{\prime}\right)$, atrial $\left(A^{\prime}\right)$, and systolic $(S)$ velocities were measured. The $E / E^{\prime}$ ratio of early mitral flow velocity $(E)$ to early diastolic velocity of the 
mitral annulus $\left(E^{\prime}\right)$ was calculated. The Doppler-derived index of combined systolic and diastolic myocardial performance (Tei index or myocardial performance index according to Tei (MPI)), defined as the sum of IVRT and isovolumetric contraction time (ICT) divided by ejection time (ET), was used for quantification of the global left ventricular function [14]. All Doppler signals were recorded at a speed of $50 \mathrm{~mm} / \mathrm{s}$. For each parameter, the average of 3 cycles was used.

\section{Statistical analysis}

Values are given as median, minimal, and maximal value in parentheses. Differences between the patient and control group were evaluated using Mann-Whitney $U$ test. Twosided $P$ values $\leq 0.05$ are considered significant. Potential associations of cumulative dose, gender, age at diagnosis, and duration of follow-up were explored using univariate and multiple regression techniques. Calculations were performed with the Statistical Package for Social Science version 12 and 15 statistical software program.

If no normal range has been defined in the literature for a given variable in this age group, a value of a given variable in the patient group was considered as abnormal if $>[\mathrm{Q} 3+$ $1.5(\mathrm{Q} 3-\mathrm{Q} 1)]$ or $<[\mathrm{Q} 1-1.5(\mathrm{Q} 3-\mathrm{Q} 1)]$ of this variable in the control group, conform the definition of an outlier in the box-and-whisker plot used to illustrate the comparison of a continuous variable between two independent groups.

\section{Results}

\section{Patient characteristics}

Characteristics of the complete cohort and the study patients are summarized in Table 1. The study patients reflect the complete cohort concerning sex distribution, age at diagnosis, age at time of study, duration of follow-up since completion of anthracycline treatment and anthracycline dose. Thirty seven adolescents and young adults, 19 males and 18 females, were examined. The median age at the time of diagnosis of the leukemia and onset of treatment was 4.8 years (range $0.2-12.2$ years). The median age at the time of the study was 19 years (range 11.6-26.8 years). The patients were examined at a median of 13.3 years (range 10.6-18.3 years) after completion of the anthracycline treatment. Nineteen patients received a cumulative anthracycline dose of $180 \mathrm{mg} / \mathrm{m}^{2}$ and 18 of $240 \mathrm{mg} / \mathrm{m}^{2}$. Nine patients received prophylactic cranial irradiation, one patient developed growth hormone deficiency, no patient received growth hormone treatment.

\section{Clinical evaluation}

None of the patients had cardiopulmonary symptoms at the time of assessment, so all were in NYHA I. The patients and controls were comparable with respect to age, sex, body surface area, body mass index, and systolic blood pressure. Male patients had a significantly and female patients a nearly significantly higher diastolic blood pressure (Table 2).

\section{Twelve-lead ECG}

No patients showed ECG abnormalities, the QTc interval was $<0.44 \mathrm{~s}$ in all patients.

Left ventricular dimensions and systolic function

Left ventricular wall dimensions and end-systolic wall stress were comparable between patients and controls but the LVPW thickening was significantly reduced in male patients (Table 3).

The shortening fraction and the ejection fraction were significantly lower in the male patient group but not in
Table 1 Characteristics of complete cohort and study patients

NS non significant

${ }^{\text {a }}$ Test type: nonparametric test, two independent samples (MannWhitney $U$ )

\begin{tabular}{lccc}
\hline & Complete cohort & Study patients & $P$ value $^{\mathrm{a}}$ \\
\hline Patients, $N$ & 85 & 37 & \\
Female sex, $N(\%)$ & $43(50.6)$ & $16(43.2)$ & $\mathrm{NS}$ \\
Age at diagnosis $<4$ years, $N(\%)$ & $35(41.2)$ & $15(40.5)$ & $\mathrm{NS}$ \\
Age at diagnosis (years) & $4.8(0.2-13.7)$ & $4.8(0.2-12.2)$ & $\mathrm{NS}$ \\
Median (range) & $19.8(11.6-28.4)$ & $19.0(11.6-26.8)$ & $\mathrm{NS}$ \\
Age at time of study (years) & $13.9(10.6-18.8)$ & $13.3(10.6-18.3)$ & $\mathrm{NS}$ \\
Median (range) & $51(60)$ & $19(51.4)$ & $\mathrm{NS}$ \\
$\begin{array}{l}\text { Years since anthracycline treatment } \\
\text { Median (range) }\end{array}$ & $34(40)$ & $18(48.6)$ & $\mathrm{NS}$ \\
Anthracycline dose & & \\
$180 \mathrm{mg} / \mathrm{m}^{2}, N(\%)$ & & & \\
$240 \mathrm{mg} / \mathrm{m}^{2}, N(\%)$ &
\end{tabular}


Table 2 Clinical characteristics of patients and controls

\begin{tabular}{|c|c|c|c|c|c|c|}
\hline & \multicolumn{3}{|l|}{ Males } & \multicolumn{3}{|l|}{ Females } \\
\hline & $\begin{array}{l}\text { Patients } \\
N=19\end{array}$ & $\begin{array}{l}\text { Controls } \\
N=16\end{array}$ & $P$ value & $\begin{array}{l}\text { Patients } \\
N=18\end{array}$ & $\begin{array}{l}\text { Controls } \\
N=14\end{array}$ & $P$ value \\
\hline Age (years) & $18.8(11.6-22.6)$ & $17.4(12.9-25.5)$ & 0.297 & $19.8(15.6-26.8)$ & $17.5(14.9-30.0)$ & 0.382 \\
\hline $\operatorname{BSA}\left(m^{2}\right)$ & $1.84(1.53-2.28)$ & $1.81(1.37-2.07)$ & 0.947 & $1.66(1.47-1.97)$ & $1.65(1.47-1.87)$ & 0.482 \\
\hline BMI $\left(\mathrm{kg} / \mathrm{m}^{2}\right)$ & $20.7(18.6-30.7)$ & $20.5(17.0-25.3)$ & 0.716 & $20.8(16.5-27.4)$ & $20.4(16.5-29.4)$ & 0.425 \\
\hline Systolic blood pressure $(\mathrm{mmHg})$ & $124(104-140)$ & $128(106-146)$ & 0.258 & $113(100-131)$ & $123(98-137)$ & 0.107 \\
\hline Diastolic blood pressure (mmHg) & $70(45-79)$ & $58(51-72)$ & 0.001 & $70(50-84)$ & $60(51-78)$ & 0.058 \\
\hline
\end{tabular}

Values are expressed as median (range); significant $P$ values $<0.05$ in bold

the female patient group. If the SF is defined as abnormal if $<28 \%$, none of the patients has an abnormal value but if defined as abnormal if $<30 \%$, three of the 37 patients $(8.1 \%)$ have an abnormal value. If the EF is defined as abnormal if $<55 \%$, the two male patients with a SF between $28 \%$ and $29 \%$ have an abnormal value (54.6\% and 54.7\%).
The reduction in LV shortening in male ALL survivors was accounted for by an increase in LVESWS (Pearson correlation coefficient -0.59 with $p>0.001$ and Spearman correlation coefficient -0.6 with $p>0.001)$. The reduction in LV shortening was also related to the reduced LVPW thickening (Pearson correlation coefficient +0.37 with

Table 3 Comparison of left ventricular dimension, systolic function, diastolic function, and time intervals between patients and controls

\begin{tabular}{|c|c|c|c|c|c|c|}
\hline & \multicolumn{3}{|l|}{ Males } & \multicolumn{3}{|l|}{ Females } \\
\hline & Patients & Controls & $P$ value & Patients & Controls & $P$ value \\
\hline \multicolumn{7}{|c|}{ Left ventricular dimensions } \\
\hline LVIDD $(\mathrm{cm})$ & $5.28(4.76-6.22)$ & $5.32(4.68-6.04)$ & 0.843 & $4.72(4.26-5.19)$ & $4.82(3.80-5.76)$ & 0.802 \\
\hline LVIDS (cm) & $3.46(3.03-4.13)$ & $3.42(2.91-3.78)$ & 0.260 & $3.08(2.60-3.31)$ & $3.06(2.38-3.51)$ & 0.706 \\
\hline LVPWD (cm) & $0.66(0.51-0.82)$ & $0.57(0.50-0.74)$ & 0.064 & $0.58(0.47-0.75)$ & $0.53(0.40-0.72)$ & 0.062 \\
\hline LVPWS (cm) & $1.15(0.92-1.46)$ & $1.18(1.00-1.34)$ & 0.842 & $1.13(0.87-1.35)$ & $1.12(0.86-1.34)$ & 0.785 \\
\hline LVPWth (\%) & $45.8(27.2-52.4)$ & $48.7(37.6-59.0)$ & 0.016 & $50.0(38.5-57.1)$ & $52.1(39.5-61.2)$ & 0.075 \\
\hline LVESWS $\left(\mathrm{g} / \mathrm{cm}^{2}\right)$ & $98.5(69.5-118.7)$ & $86.7(69.2-125.5)$ & 0.640 & $78.1(54.7-107.9)$ & $85.1(56.9-110.4)$ & 0.405 \\
\hline LVmass (g) & $132(87-184)$ & $121(93-188)$ & 0.196 & $95(67-144)$ & $85(60-152)$ & 0.217 \\
\hline LVmass Index $\left(\mathrm{g} / \mathrm{m}^{2}\right)$ & $71(56-105)$ & $66(53-91)$ & 0.233 & $56(43-73)$ & $52(36-81)$ & 0.267 \\
\hline \multicolumn{7}{|l|}{ Systolic function } \\
\hline SF $(\%)$ & $34.0(28.6-39.4)$ & $36.8(31.6-40.1)$ & 0.009 & $36.9(30.4-40.8)$ & $37.6(31.1-44.4)$ & 0.706 \\
\hline $\mathrm{EF}(\%)$ & $61.3(54.6-70.5)$ & $66.1(59.2-70.3)$ & 0.014 & $66.6(58.6-71.6)$ & $67.2(58.8-76.1)$ & 0.738 \\
\hline \multicolumn{7}{|c|}{ Diastolic function mitral valve and tissue Doppler imaging } \\
\hline E maximum $(\mathrm{m} / \mathrm{s})$ & $0.78(0.64-0.96)$ & $0.78(0.59-1.11)$ & 0.817 & $0.85(0.48-1.01)$ & $0.88(0.61-1.03)$ & 0.210 \\
\hline A maximum $(\mathrm{m} / \mathrm{s})$ & $0.46(0.30-0.63)$ & $0.36(0.24-0.49)$ & 0.002 & $0.46(0.31-0.67)$ & $0.45(0.28-0.77)$ & 0.403 \\
\hline$E / A$ maximum & $1.83(1.34-2.66)$ & $2.02(1.66-3.27)$ & 0.003 & $1.69(0.74-2.89)$ & $2.07(1.27-3.11)$ & 0.160 \\
\hline $\mathrm{E}^{\prime}(\mathrm{cm} / \mathrm{s})$ & $13.8(9.4-17.3)$ & $15.0(10.1-18.8)$ & 0.262 & $14.1(9.9-19.3)$ & $13.6(12.0-15.4)$ & 0.857 \\
\hline $\mathrm{E} / \mathrm{E}^{\prime}$ & $5.71(4.65-7.53)$ & $5.44(3.61-7.99)$ & 0.270 & $5.74(4.47-8.25)$ & $6.61(4.00-8.50)$ & 0.060 \\
\hline $\mathrm{A}^{\prime}(\mathrm{cm} / \mathrm{s})$ & $7.5(5.1-9.7)$ & $7.4(4.0-11.5)$ & 0.678 & $8.1(3.9-11.5)$ & $7.4(5.7-10.0)$ & 0.706 \\
\hline $\mathrm{S}(\mathrm{cm} / \mathrm{s})$ & $8.8(7.5-11.0)$ & $9.5(6.7-12.9)$ & 0.156 & $9.4(8.1-15.2)$ & $8.6(7.4-10.2)$ & 0.081 \\
\hline \multicolumn{7}{|l|}{ Time intervals } \\
\hline Tei index left ventricle & $0.55(0.42-0.68)$ & $0.44(0.38-0.49)$ & $<0.001$ & $0.54(0.40-0.81)$ & $0.43(0.38-0.52)$ & 0.002 \\
\hline IVRT (ms) & $75(55-98)$ & $63(53-75)$ & $<0.001$ & $80(53-95)$ & $60(45-82)$ & 0.003 \\
\hline ICT (ms) & $83(55-115)$ & $60(42-68)$ & 0.001 & $70(55-143)$ & $62(33-81)$ & 0.053 \\
\hline ET (ms) & $288(245-332)$ & $283(252-310)$ & 0.398 & $281(227-340)$ & $276(262-300)$ & 0.544 \\
\hline
\end{tabular}

Values are expressed as median (range); significant $P$ values $<0.05$ in bold 
$p=0.027$ and Spearman correlation coefficient +0.37 with $p=0.030$ ).

\section{Diastolic function}

\section{Conventional Doppler measurements}

The mitral ( $V$ maximum) A-wave velocity was significantly higher in the male patient group with consequently significantly lower early filling velocity to atrial contraction velocity $(E / A)$ ratio. This could not be demonstrated in the female patient group. In none of the male patients the $E / A$ ratio was abnormal compared to the normal range in the male controls (Table 3).

\section{Tissue Doppler}

Tissue Doppler-derived values of the septal mitral annulus were comparable between patients and controls (Table 3).

\section{Time intervals and duration}

The Tei index for the left ventricle was significantly higher in both male and female patient group due to a significantly longer IVRT and a longer ICT which was significantly longer in males and nearly significantly longer in females. Ten of the 19 male and Two of the 16 female patients have an abnormal Tei index compared to the normal range in the controls. Eight of the 19 male and three of the 16 female patients have an abnormal IVRT compared to the normal range of the controls (Table 3).

\section{Correlation analysis}

We could not find any correlation between cumulative anthracycline dose, age at diagnosis, and duration of follow-up and the different cardiac parameters in the patient group. Echocardiographic abnormalities were found more frequently in the male ALL survivors but the figures for the sex difference did not reach statistical significance.

\section{Discussion}

In this study no clinical relevant late cardiac damage was demonstrated in long-term survivors of childhood ALL who received 180 or $240 \mathrm{mg} / \mathrm{m}^{2}$ of anthracyclines 13.3 years earlier. Subclinical cardiac abnormalities were present with a prevalence varying depending on the parameters used to address this topic.

Anthracyclines impair the growth potential of the myocardium. Myocardial histology suggests that surviving myocytes compensate to maintain normal cardiac structure by hypertrophy.

Non-adequate myocyte growth in the years after treatment finally causes a thinning of the wall of the left ventricle, development of inappropriate afterload with in some cases chamber dilatation and the development of cardiomyopathy. Impairment of myocyte growth of the left ventricular myocardium can be seen on echocardiography as a thinner left ventricular wall with reduced left ventricular wall thickness and mass $[2,4,15,16]$. We did not find reduced left ventricular dimensions or mass which can be explained by the lower doses of anthracyclines our patients received. The cumulative dose of anthracyclines is the most important factor for cardiotoxicity in the previous studies. Although a cumulative dose $<250 \mathrm{mg} / \mathrm{m}^{2}$ is said to be reasonably safe, it has also been shown that no dose is completely safe $[4,5$, $16,17]$. LV shortening, as determined by SF, was reduced in male ALL survivors as compared to the healthy controls but was still in the normal range with a $\mathrm{SF}<28 \%$ considered as abnormal. If the SF is defined as abnormal if $<30 \%$, three of the 37 patients $(8 \%)$ have an abnormal value. Like others, we documented that when the SF was abnormal, this was accounted for by a reduced LVPW thickening and an increase in LVESWS.

Diastolic changes have been found to precede systolic dysfunction. Impaired relaxation has been proposed as a consequence of chronic progressive myocardial damage induced by anthracyclines [7, 8]. In this study, LV diastolic performance was assessed from pulsed wave mitral Doppler flow and tissue Doppler imaging. In the male ALL survivors, the mitral ( $V$ maximum) A-wave velocity was significantly higher with consequently significantly lower early filling velocity to atrial contraction velocity $(E / A)$ ratio but in none of the patients the $E / A$ ratio was abnormal compared to the normal range of the controls. Early filling velocity was comparable between patients' end controls. Since mitral flow velocities are dependent on load present during filling, LV diastolic performance was also assessed from tissue Doppler imaging by measuring $E^{\prime}$ as a parameter of diastolic function independent of left ventricular filling. Although $E^{\prime}$ was lower in the male patients as compared to the male controls, this difference did not reach statistical significance. In general, $E^{\prime}$ was comparable between patients and controls as was $E / E^{\prime}$. So we did not find clearly abnormal left ventricular filling abnormalities with conventional nor tissue Doppler imaging at the septal mitral annulus. The applicability of TDI for estimating subclinical myocardial dysfunction was more extensively studied by other investigators $[10,18-20]$. In contrast to our findings, they could demonstrate an added value of TDI in detecting signs of mostly subtle regional diastolic and systolic myocardial dysfunction in asymptomatic patients after exposure to low to moderate doses of anthracyclines. 
ALL survivors showed an impaired relaxation characterized by significant lengthening of the isovolumetric relaxation time. In $30 \%$ of the patients, the IVRT was abnormal compared to the normal range of the controls. IVRT is not only function of the rate of isovolumic relaxation but also of the pressure gradient between the pressure in the aorta just preceding closure of the aortic valve and the pressure in the atrium upon opening of the mitral valve. The possible influence of an altered pressure gradient was assessed by measuring systolic blood pressure and aortic pulse wave velocity which are related to left ventricular afterload and which were comparable between patient and control group. This was also the case for the normalized pulmonary acceleration time which reflects pulmonary arterial pressure and, indirectly, left atrial pressure. Isovolumic relaxation is an active process vulnerable to anthracycline toxicity. We suggest isovolumic relaxation to be impaired due to reduced left ventricular compliance being the first sign of systolic dysfunction.

Global left ventricular function was assessed by using the Tei index or MPI. The Tei index was significantly increased in the patient group. In comparison with the normal range of the control group, $32 \%$ of the patients have an abnormal Tei index. This is due to a significantly longer IVRT and a nearly significantly longer ICT. The Tei index has been described as a sensitive parameter for detection of subclinical myocardial dysfunction. Several other authors found an increased MPI as indicator of subclinical damage in childhood cancer survivors after treatment with a low to median dose of anthracyclines [17, 20,21], and in two of these studies, also due to a significantly prolonged IVRT $[17,20]$.

We could not demonstrate a correlation with anthracycline dose probably because both doses are low doses. We did not find female gender or a young age at treatment, especially younger than 4 years, to be a risk factor. We suggest that this is related to the fact that the previously found correlation is weaker in the low cumulative anthracycline dose range. This can be deduced from the curves about the probability of late depressed fractional shortening in relation to cumulative anthracycline dose and age at the time of diagnosis and about the probability of late depressed contractility in relation to cumulative anthracycline dose and sex provided by Lipshultz et al.[22]. On the contrary, we found echocardiographic abnormalities more frequently in male ALL survivors although the figures for the sex difference did not reach statistical significance. We do not think male ALL survivors to be more prone to develop late anthracycline cardiotoxicity. This is a finding from a small cohort that needs further study.

We could not demonstrate a correlation with longer duration of follow-up. It is known that the risk of cardiac abnormalities increases with time since anthracycline exposure for patients who received a cumulative dose $>250 \mathrm{mg} / \mathrm{m}^{2}$
$[16,23,24]$ but this is not yet known for patients who received a lower cumulative dose.

The small cohort of survivors is the main limitation of our study. This causes less power in the statistical analysis. The findings need further study to verify in a large cohort.

On the other hand data concerning the cardiovascular status of survivors more than 10-15 years after completion of treatment with cumulative doses in the range of 180 $240 \mathrm{mg} / \mathrm{m}^{2}$ are still limited. These data are important since the majority of the long-term survivors of childhood cancer exposed to anthracyclines, received a cumulative dose $<250 \mathrm{mg} / \mathrm{m}^{2}$.

In conclusion, at a median of 13.3 years after exposure to a cumulative dose of anthracyclines of 180 or $240 \mathrm{mg} / \mathrm{m}^{2}$, no clinical relevant cardiotoxicity was found but subclinical cardiac abnormalities were present in up to one third of the patients. Three of the 37 patients ( $8 \%$ ) have an SF $<30 \%$ and 11 of the 37 patients ( $30 \%$ ) have a prolonged IVRT compared to the normal range of the controls. The prognostic significance of the changes in these cardiac parameters is still not well-known. Considering the growing knowledge of the mechanisms of cardiotoxicity which can continue long after the end of treatment $[25,26]$ and the lack of data of followup on the very long-term, continuous follow-up of these survivors is needed. Guidelines concerning cardiac monitoring were recently developed by the cardiovascular disease task force of the Children's Oncology Group [27]. Also after exposure to a low dose of antracyclines, survivors should be encouraged to follow a preventive heart healthy lifestyle and monitored lifelong.

Open Access This article is distributed under the terms of the Creative Commons Attribution Noncommercial License which permits any noncommercial use, distribution, and reproduction in any medium, provided the original author(s) and source are credited.

\section{References}

1. Goorin AM, Chauvenet AR, Perez-Atayde AR, et al. Initial congestive heart failure, six to ten years after doxorubicin chemotherapy for childhood cancer. J Pediatr. 1990;116:144-7.

2. Lipshultz SE, Colan SD, Gelber RD, et al. Late cardiac effects of doxorubicin therapy for acute lymphoblastic leukemia in childhood. N Engl J Med. 1991;324:808-15.

3. Steinherz LJ, Steinherz P, Tan CTC, et al. Cardiac toxicity 4 to 20 years after completing anthracycline therapy. JAMA. 1991;266:1672-7.

4. Sorensen K, Levitt G, Sebag-Montefiore D, et al. Anthracycline dose in childhood acute lymphoblastic leukemia: Issues of early survival versus late cardiotoxicity. J Clin Oncol. 1997;15:61-8.

5. Nysom K, Holm K, Lipsitz SR, et al. Relationship between cumulative anthracycline dose and late cardiotoxicity in childhood acute lymphoblastic leukemia. J Clin Oncol. 1998;16:545-50.

6. Rammeloo LAJ, Postma A, Soboka-Plojhar MA. Low-dose daunorubicin in induction treatment of childhood acute lympho- 
blastic leukaemia: no long-term cardiac damage in a randomised study of the Dutch childhood leukaemia study group. Med Ped Oncol. 2000;35:136-9.

7. De Wolf D, Suys B, Maurus R, et al. Dobutamine stress echocardiography in the evaluation of late anthracycline cardiotoxicity in childhood cancer survivors. Pediatr Res. 1996;39:504-12.

8. Dorup I, Levitt G, Sullivan I, Sorensen K. Prospective longitudinal assessment of late anthracycline cardiotoxicity after childhood cancer: the role of diastolic function. Heart. 2004;90:1214-16.

9. Suys BE, Katier N, Rooman RP, et al. Female children and adolescents with type 1 diabetes have more pronounced early echocardiographic signs of diabetic cardiomyo-pathy. Diabetes Care. 2004;27:1947-53.

10. Kapusta L, Thijssen JM, Groot-Loonen J, et al. Tissue Doppler Imaging in detection of myocardial dysfunction in survivors of childhood cancer treated with anthracyclines. Ultrasound Med Biol. 2000;26(7):1099-108.

11. Vilmer E, Suciu S, Ferster A, et al. Long-term results of three randomized trials $(58831,58832,58881)$ in childhood acute lymphoblastic leukemia: a CLCG-EORTC Report. Children Leukemia Cooperative Group. Leukemia. 2000;14(12):2257-66.

12. Rowland DG, Gutgesell HP. Use of mean arterial pressure for noninvasive determination of left ventricular end-systolic wall stress in infants and children. Am J Cardiol. 1994;4:98-9.

13. Devereux RB, Lutas EM, Casale PN, et al. Standardization of Mmode echocardiographic left ventricular anatomic measurements. J Am Coll Cardiol. 1984;4:1222-30.

14. Tei C. New noninvasive index for combined systolic and diastolic ventricular function. J Cardiol. 1995;26:135-6.

15. Sorensen K, Levitt GA, Bull C, et al. Late anthracycline cardiotoxicity after childhood cancer. A prospective longitudinal study. Cancer. 2003;97:1991-8.

16. Lipshultz SE, Lipsitz SR, Sallan SE, et al. Chronic progressive cardiac dysfunction years after doxorubicin therapy for childhood acute lymphoblastic leukemia. J Clin Oncol. 2005;23:2629-36.
17. Elbl L, Hrstkova H, Chaloupka V. The late consequences of anthracycline treatment on left ventricular function after treatment of childhood cancer. Eur J Pediatr. 2003;162(10):690-6.

18. Stapleton GE, Stapleton LC, Martinez A, et al. Evaluation of longitudinal ventricular function with tissue Doppler echocardiography in children treated with anthracyclines. J Am Soc Echocardiogr. 2007;20:492-7.

19. Rathe M, Carlsen NLT, Oxhøj H. Late cardiac effects of anthracycline containing therapy for childhood lymphoblastic leukemia. Pediatr Blood Cancer. 2007;48:663-7.

20. Ganame J, Cluas P, Uyttebroeck A, et al. Myocardial dysfunction late after low-dose Anthracycline treatment in asymptomatic pediatric patients. J Am Soc Echocardiogr. 2007;20:1351-8.

21. Eidem BW, Sapp BG, Suarez CR, et al. Usefulness of the Myocardial Performance Index for early detection of anthracycline-induced cardiotoxicity in children. Am J Cardiol. 2001;87:1120-2.

22. Lipshultz SE, Lipsitz SR, Mone SM, et al. Female sex and drug dose as risk factors for late cardiotoxic effects of doxorubicin therapy for childhood cancer. N Engl J Med. 1995;332:1738-43.

23. Pein F, Sakiroglu O, Dahan M, et al. Cardiac abnormalities 15 years and more after Adriamycin therapy in 229 childhood survivors of a solid tumour at the Institut Gustave Roussy. Br J Cancer. 2004;91:37-44.

24. van Dalen EC, van der Pal HJ, Kok WE, et al. Clinical heart failure in a cohort af children treated with anthracyclines: a longterm follow-up study. Eur J Cancer. 2006;42:3191-8.

25. Wouters KA, Kremer LCM, Miller TL, et al. Protecting against anthracycline-induced Myocardial damage: a review of the most promising strategies. Br J Haematol. 2005;131:561-78.

26. Lipshultz SE, Alvarez JA, Scully RE. Anthracycline associated cardiotoxicity in survivors of childhood cancer. Heart. 2008;94:525-33.

27. Shankar SM, Marina N, Hudson MM, et al. Monitoring for cardiovascular disease in survivors of childhood cancer: report from the carciovascular disease task force of the Children's Oncology Group. Pediatrics. 2008;121:e387-96. 